\title{
A rubust fractional order PID controller for MIMO power systems
}

\author{
Vasampalli Shashidhar ${ }^{1}$, and Dola Gobinda Padhan ${ }^{2, *}$ \\ ${ }^{1,2}$ Gokaraju Rangaraju Institute of Engineering and Technology, Hyderabad, India
}

\begin{abstract}
This paper proposes a novel fractional order PID control scheme for Multi Input and Multi Output (MIMO) power systems. This control scheme utilizes Cuckoo Search algorithm to tune the fractional PID controller to guarantee better closed loop performance in the transmission or distribution networks. Cuckoo Search optimization algorithm is proposed to optimize gain values of the fractional PID controller. The effectiveness of the proposed control strategy has been assessed by simulations in MATLAB/Simulink platform. The robustness of the proposed controller has been validated by varying the plant parameters.
\end{abstract}

\section{Introduction}

In the MIMO power systems there are a number of control areas and each control area is obliged to generate power to cover the customers load requirements as well as maintaining the systems frequency and the interchanged power at the scheduled values. Therefore, load frequency controller (LFC) [1], is designed and implemented to automatically balance generated power and load demand in each control area so that the quality of the power delivered is maintained at the requisite level. The analysis and design of load frequency control system of individual generator eventually controlling large interconnections between different control areas plays a vital role in automation of power system. The purpose of LFC is to maintain system frequency very close to a specified nominal value to maintain generation of individual units at the most economical value and to keep the correct value of the line power between different control areas. Many investigations in the area of LFC problem of interconnected

Cuckoo Search is a Swarm Intelligence based Metaheuristic optimization algorithm inspired by the brood parasitic behaviour observed in the cuckoo bird species [2]. Cuckoo Search has been shown to be superior to other algorithms such as GA and PSO in finding a global optimum of many numeric test functions. Cuckoo Search has also been applied to many structural designing optimization problems such as design of wind turbines, vehicular components and welding structures [3]. Cuckoo Search has also been proposed for machine learning techniques like Feed Forward Neural Network Training and Document Clustering
This paper is organized as follows: section 2 presents four area power systems. Section 3 introduces fractional order controller. Section 4 presents Cuckoo Search algorithm. In section 5, simulation results have been discussed in details. Section 6 presents the conclusion.

\section{Four Area Power Systems}

A four area power system comprises four areas that are interconnected by high voltage transmission lines or tielines. The trend of frequency measured in each control area is an indicator of the trend of the mismatch power in the interconnection and not in the control area alone [4]. Parameter uncertainties, model mismatch and unexpected external disturbances are the key challenges for controller design while dealing with LFC issue of power systems. The simple block diagram of four areas interconnected power systems is shown in Fig. 1. Also, the general block diagram representation of control area ' $i$ ' of MIMO power systems is shown in Fig. 2. Here, reheat turbine has been considered for Area 1, Area 2 and Area 3. For Area 4, non-reheat turbine is considered for simulation.

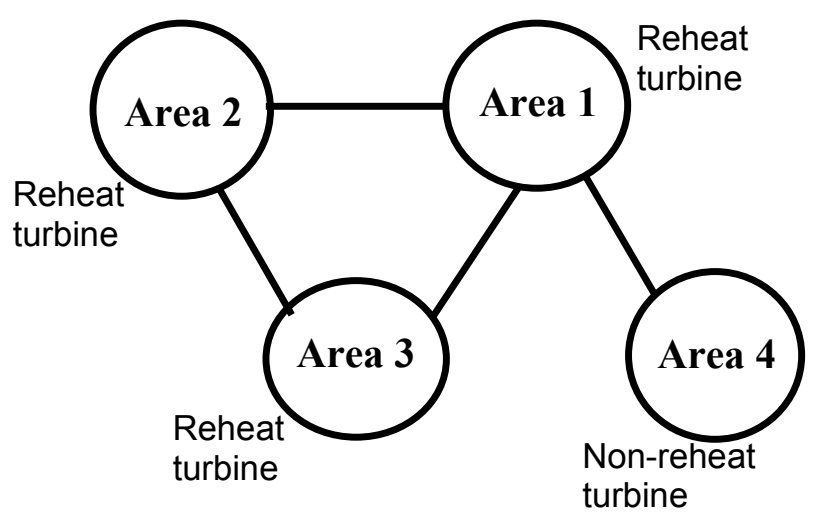


Fig. 1. Simplified Block diagram of 4 areas inter-connected power systems

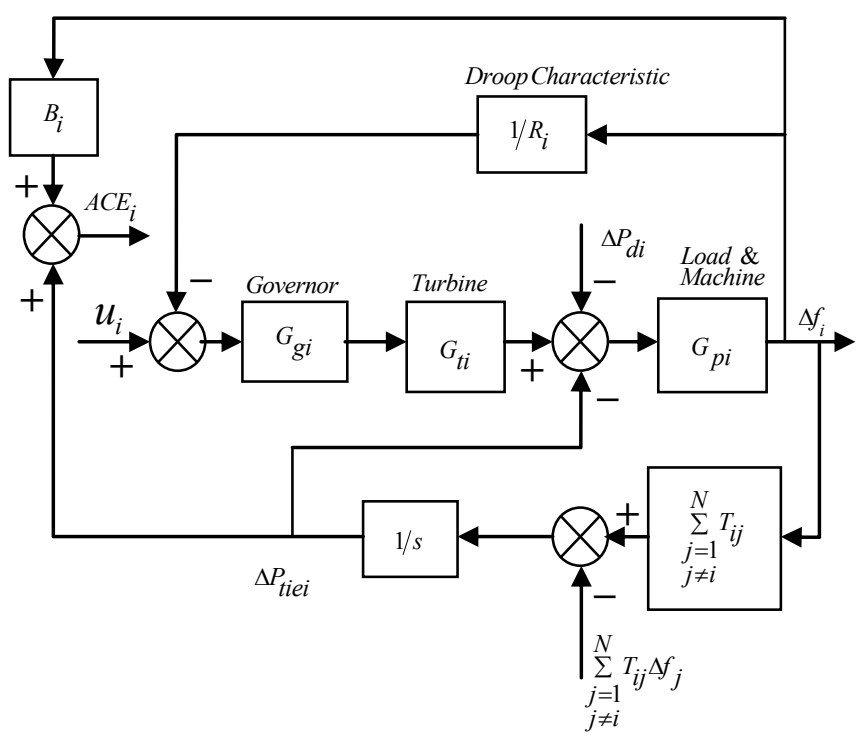

Fig. 2. General Block diagram representation of control area $i$ of MIMO Power systems

For interconnected power systems, the governor dynamics is given by

$$
G_{g}=\frac{1}{T_{g} s+1}
$$

The dynamics for non-reheat turbine is in the form

$$
G_{t}=\frac{1}{T_{t} s+1}
$$

The dynamics for reheat turbine is given by

$$
G_{t r}=\frac{c T_{r} s+1}{\left(\mathrm{~T}_{r} \mathrm{~s}+1\right)\left(\mathrm{T}_{t} \mathrm{~s}+1\right)}
$$

where is $T_{r}$ a constant and $c$ is the percentage of the power generated by the reheat process in the total generated power.

The transfer function for load and machine is

$$
G_{p}=\frac{K_{p}}{T_{p} s+1}
$$

\section{Fractional order $P I^{\lambda} D^{\mu}$ controller}

From the literature it is confirmed that, the fractional controller outperforms the integer-order PID Controller.
The control action of the $P I^{\lambda} D^{\mu}$ controller can be expressed in time domain as

$$
u(\mathrm{t})=\mathrm{K}_{p} e(\mathrm{t})+\mathrm{K}_{i} D^{-\lambda} e(\mathrm{t})+\mathrm{K}_{d} D^{\mu} e(\mathrm{t})
$$

Where $e(t)$ is error signal. By applying Laplace transform to equation (5) with zero initial conditions, the following equation is obtained

$$
G_{c}(\mathrm{~s})=\mathrm{K}_{p}+\frac{K_{i}}{s}+K_{d} s
$$

Several approaches are suggested for tuning of fractional PID controllers over the years [5-6]. However, there is still no well-established, general tuning algorithm.

Nevertheless, optimization techniques may be applied to the tuning problem instead. The parameter set to optimize consists of fractional PID parameters such as $\left(\mathrm{K}_{\mathrm{p}}, \mathrm{K}_{\mathrm{i}}, \mathrm{K}_{\mathrm{d}}, \lambda\right.$ and $\mu$ ). Cuckoo Search algorithm has fewer control parameters to fine tune than other algorithms. Moreover, tweaking of control parameters for a particular problem is not needed [2]. Hence Cuckoo search is more generic and robust for many optimization problems.

The integral time absolute error $\left(\right.$ ITAE $\left.=\int_{0}^{t} t|e(\mathrm{t})| d t\right)$

performance criteria has been used as objective functions for optimization .

\section{Cuckoo search algorithm}

Cuckoo Search is a nature inspired optimization algorithm based on the cuckoo bird's parasitic breeding behaviour of laying its eggs in the nests of different bird species. The cuckoo bird deposits her eggs in the nest of a host bird whose eggs closely resemble her own eggs and uses the services of host birds to hatch her own eggs. The cuckoo search algorithm simulates the cuckoo bird's intelligent search strategy of finding the best host nest to deposit her egg.

The cuckoos make series of flights searching and evaluating one nest after the other until they find the optimal host nest to spawn their eggs. The series of flights the cuckoos make can be classified into two types namely Lévy/Global Flight and Local Flight. The Lévy Flight is based on the Lévy Probability distribution. Many animals and insects forage using the Lévy flight for finding their prey/food location [3]. During the Local Flight, the cuckoo examines around the host nests which the other cuckoos are using, and finds the fittest nest among them and computes the distance and direction of her Local flight based on that. The flow chart for Cuckoo search algorithm is shown in Fig. 3. 


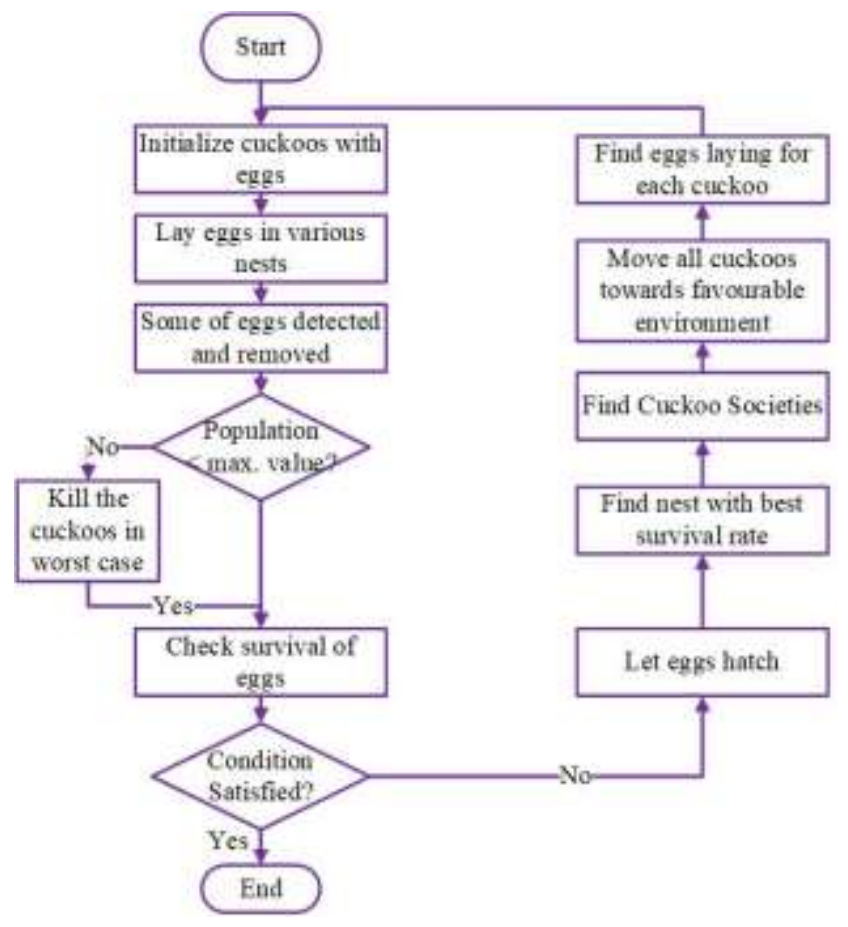

Fig. 3. Flow chart for Cuckoo search algorithm

\section{Simulation Results}

In the simulation results, area 1 , area 2 and area 3 are considered as the area with reheat thermal turbine, and area 4 is represented as the area with non-reheat turbine.

The turbine dynamics of area 1 , area 2 and area 3 as studied by [4] are considered.

The governer dynamics of area 1 , area 2 and area 3 is given by $G_{g}=1 /(0.2 \mathrm{~s}+1)$

The load and machine dynamics of area 1, area 2 and area 3 is $G_{p}=120 /(20 s+1)$

The turbine dynamics of area 1 , area 2 and area 3 is given

$$
G_{t r}=(0.333 * 20 s+1) /((20 s+1)(0.3 s+1))
$$

The governer dynamics of area 4 is given by $G_{g n r}=1 /(0.08 \mathrm{~s}+1)$

The load and machine dynamics of area 4 is $G_{p n r}=120 /(20 s+1)$

The turbine dynamics of area 4 is given by $G_{t n r}=1 /(0.3 \mathrm{~s}+1)$

And for all cases droop parameter, $\mathrm{R}=2.4$

The synchronising constants are 0.0707 and the frequency bias constants are 0.425 .

Using Cuckoo search algorithm, the parameters of the controller for area 1 , area 2 and area 3 are $\mathrm{K}_{\mathrm{p}}=1.1895$, $\mathrm{K}_{\mathrm{i}}=0.6231, \mathrm{~K}_{\mathrm{d}}=0.6487, \lambda=0.99$ and $\mu=0.78$. Also the parameters of the controller for area 4 are $\mathrm{K}_{\mathrm{p}}=1.9822, \mathrm{~K}_{\mathrm{i}}=0.5242, \quad \mathrm{~K}_{\mathrm{d}}=0.1756, \lambda=1.12$ and $\mu=0.82$.
To show the performance fractional order PID controller, a step load 0.01 is applied to arealat $\mathrm{t}=5 \mathrm{~s}$, area 2 at $\mathrm{t}=100 \mathrm{~s}$, area 3 at $\mathrm{t}=150 \mathrm{~s}$ and followed by a step load 0.01 to area 4 at $t=250 \mathrm{~s}$. Fig. 4 to Fig. 7 illustrates the frequency deviations of the four different areas. Fig. 8 to Fig. 11 shows the tie-line power deviations of the four areas. From the simulation results, it can be seen that the frequency errors, and tie-line power deviations have been driven to zero by proposed controller in the presences of power load changes.

In order to test the robustness of the controller, the variations of the parameters of the non-reheat and reheat turbines in the four areas are assumed to be $50 \%$ of their nominal values. However, the controller parameters are not changed with the variations of the system parameters. Fig. 4 to Fig. 7 illustrates the frequency errors of four areas with the variant parameter values. Fig. 8 to Fig. 11 shows the tie-line power errors of the four areas with the variant parameter values. From the simulation results, it can be seen that despite such large parameter variations, the system responses do not show notable difference from the nominal values. Therefore, the simulation results demonstrate the robustness of proposed controller against system parameter variations.

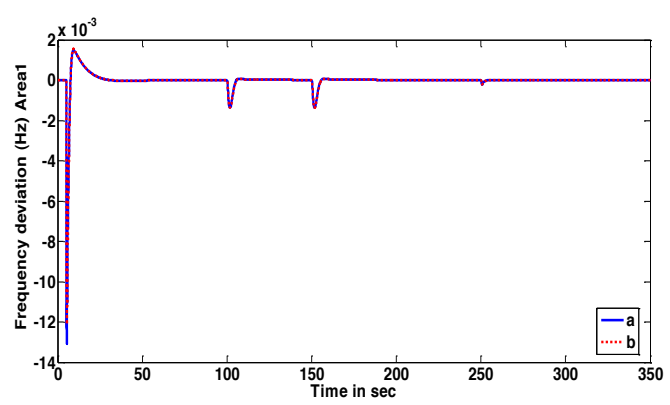

Fig. 4. Frequency deviation for Area 1, solid line-a-nominal response, dotted line-b-perturbed response

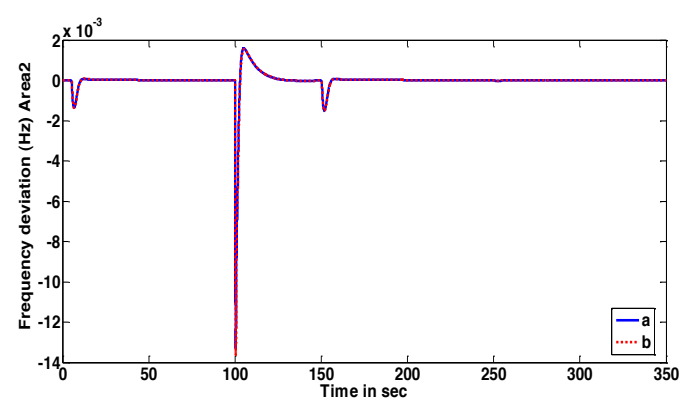

Fig. 5. Frequency deviation for Area 2, solid line-a-nominal response, dotted line-b-perturbed response 


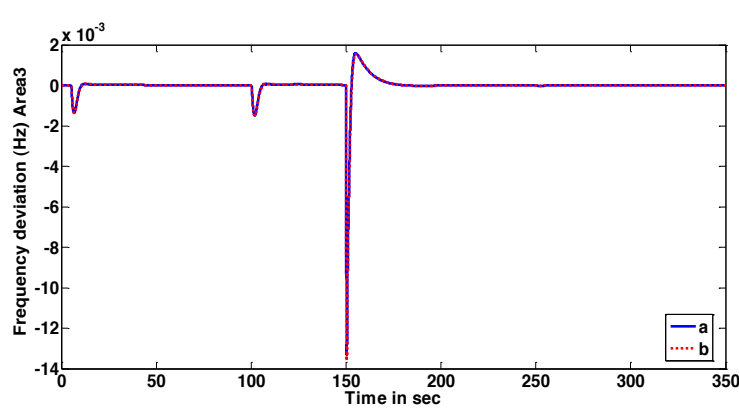

Fig. 6. Frequency deviation for Area 3, solid line-a-nominal response, dotted line-b-perturbed response

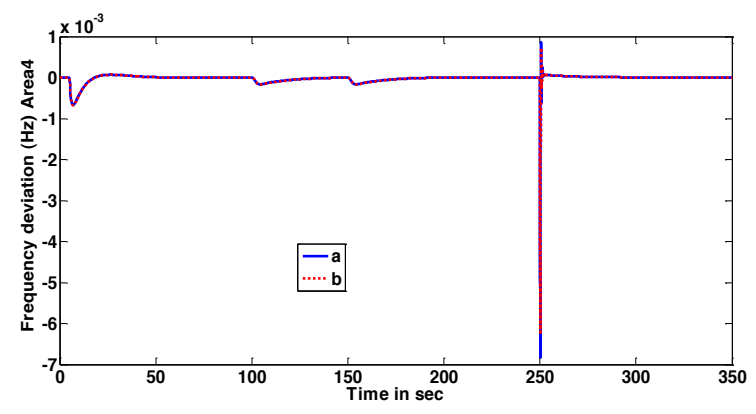

Fig. 7. Frequency deviation for Area 4, solid line-a-nominal response, dotted line-b-perturbed response

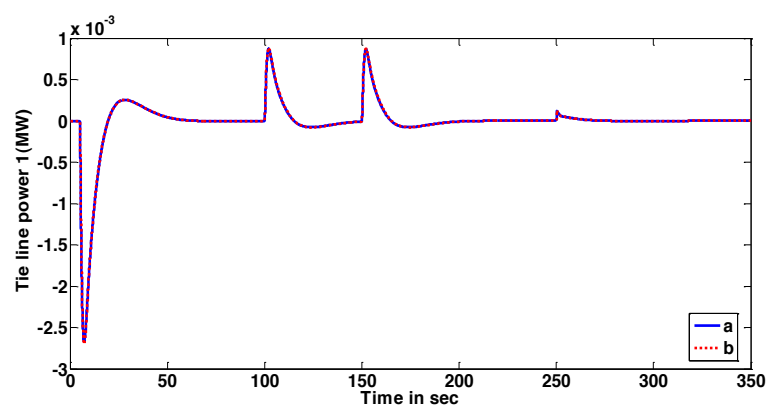

Fig. 8. Tie line power deviation for Area 1 and Area 2, solid line-a-nominal response, dotted line-b-perturbed response

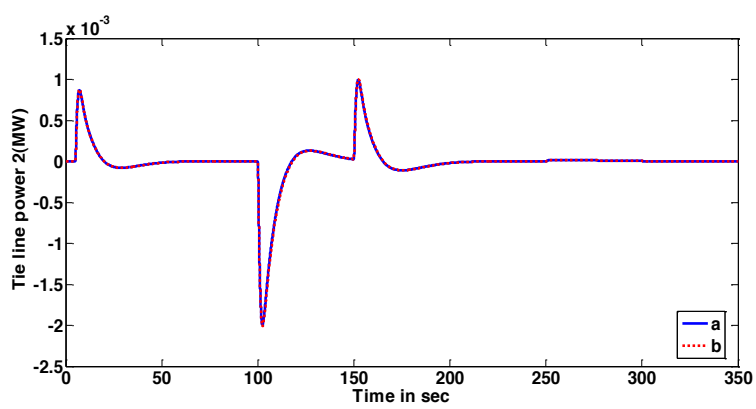

Fig. 9. Tie line power deviation for Area 2 and Area 3, solid line-a-nominal response, dotted line-b-perturbed response

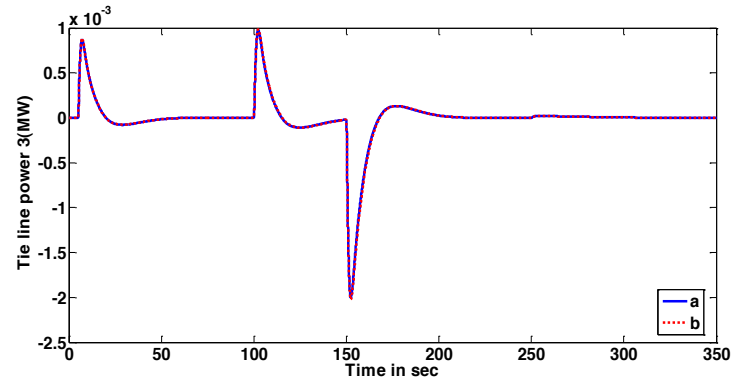

Fig. 10. Tie line power deviation for Area 3 and Area 1, solid line-a-nominal response, dotted line-b-perturbed response

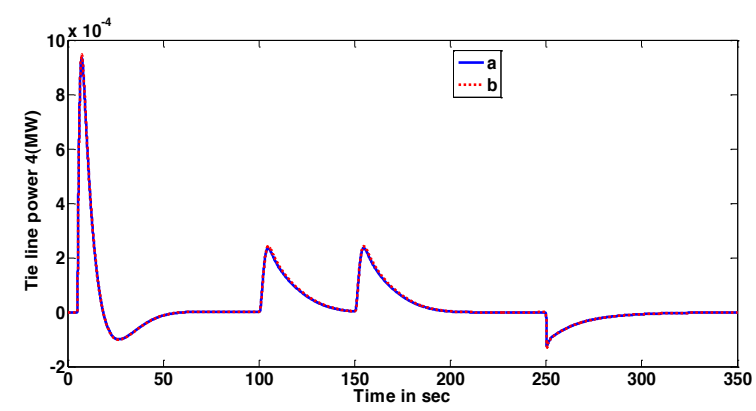

Fig. 11. Tie line power deviation for Area 4 and Area 1, solid line-a-nominal response, dotted line-b-perturbed response

\section{Conclusion}

In this paper, a fractional $P I^{\lambda} D^{\mu}$ controller is designed using Cuckoo search algorithm for load frequency control of four-area interconnected MIMO power systems. The robustness of the controllers are tested with different plant parameters uncertainty scenarios.

\section{References}

1. A. Khodabakhshian, R. Hooshmand, A new PID controller design for automatic generation control of hydro power systems, Electrical Power and Energy Systems 32, 375-382, (2010)

2. E. Valian, S. Mohanna and S. Tavakoli, 'Improved Cuckoo Search Algorithm for Feed forward Neural Network Training', International Journal of Artificial Intelligence \& Applications, 2 (3), 36-43, (2011)

3. A. Yildiz, 'Cuckoo search algorithm for the selection of optimal machining parameters in milling operations', Int J Adv Manuf Technol, 64 (1), 55-61, (2012)

4. D.G. Padhan, S. Majhi, A new control scheme for PID load frequency controller of single-area and multi-area power systems, ISA Transactions, 52, 242-251, (2013)

5. C.A. Monje, Y.Q. Chen, B.M. Vinagre, D. Xue, V. Feliu, Fractional order Systems and Controls Fundamentals and Applications, SpringerVerlag, London, (2010)

6. C.N. Zhao, D.Y. Xue, Y.Q. Chen, A fractional order PID tuning algorithm for a class of fractional order 
plants, Proceedings of the International Conference on Mechatronics and Automation. Niagara, Canada,

216-221, (2005) 